\title{
Exchange Rate Risk and the Impact of Regret on Trade *
}

\author{
Udo Broll ${ }^{\dagger}$ \\ Dresden University of Technology \\ Peter Welzel \\ University of Augsburg \\ and \\ Kit Pong Wong \\ University of Hong Kong
}

March 2014

\begin{abstract}
This paper examines the behavior of the regret-averse multinational firm under exchange rate uncertainty. Regret-averse preferences are characterized by a modified utility function that includes disutility from having chosen ex-post suboptimal alternatives. We show that the conventional results that the multinational firm optimally produces less, sells more domestically, and export less abroad under uncertainty than under certainty holds if the multinational firm is not too regret averse. Using a simple binary model wherein the random spot exchange rate can take on either a low value or a high value with positive probability, we show that the conventional results may not hold, particularly when the multinational firm is sufficiently regret averse and the low spot exchange rate is very likely to prevail.
\end{abstract}

JEL classification: D81; F23; F31

Keywords: Exchange rate risk; Multinational firms; Regret theory

\footnotetext{
${ }^{*}$ We would like to thank George Tavlas (the editor) and two anonymous referees for their helpful comments and suggestions. The usual disclaimer applies.

${ }^{\dagger}$ Corresponding author. Department of Business and Economics, School of International Studies (ZIS), Dresden University of Technology, 01062 Dresden, Germany. E-mail: udo.broll@tu-dresden.de (U. Broll).
} 


\title{
Exchange Rate Risk and the Impact of Regret on Trade
}

\begin{abstract}
This paper examines the behavior of the regret-averse multinational firm under exchange rate uncertainty. Regret-averse preferences are characterized by a modified utility function that includes disutility from having chosen ex-post suboptimal alternatives. We show that the conventional results that the multinational firm optimally produces less, sells more domestically, and export less abroad under uncertainty than under certainty holds if the multinational firm is not too regret averse. Using a simple binary model wherein the random spot exchange rate can take on either a low value or a high value with positive probability, we show that the conventional results may not hold, particularly when the multinational firm is sufficiently regret averse and the low spot exchange rate is very likely to prevail.
\end{abstract}

Keywords Exchange rate risk $\cdot$ Multinational firms $\cdot$ Regret theory

JEL Classification D81 · F23 · F31

\section{Introduction}

The study of the multinational firm under exchange rate uncertainty has been the subject of considerable research in decision making under uncertainty (Broll et al. 2009; Broll and Wong 2006; Broll and Zilcha 1992; Fillat and Garetto 2010; Mrázová and Neary 2011; Pflüger and Russek 2013; Russ 2007; Schmitz 2010; to name just a few). The extant literature examines the production and export decisions of the multinational firm using the standard von Neumann-Morgenstern expected utility representation. It is shown that the risk-averse multinational firm optimally produces less, sells more domestically, and exports less abroad when the exchange rate uncertainty prevails than when the random spot exchange rate is fixed at the expected value (say via foreign exchange forward/futures trading).

In reality, multinational firms may have desires to avoid consequences wherein ex-post suboptimal decisions appear to have been made even though these decisions are ex-ante 
optimal based on the information available at that time. To account for this consideration, Bell $(1982,1983)$ and Loomes and Sugden (1982) propose regret theory that defines regret as the disutility arising from not having chosen the ex-post optimal alternative, which is later axiomatized by Quiggin (1994) and Sugden (1993). Regret theory is supported by a large body of experimental literature that documents regret-averse preferences among individuals (see, e.g., Loomes, 1988; Loomes et al., 1992; Loomes and Sugden, 1987; Starmer and Sugden, 1993). Steil (1993) conducts a survey of 26 multinational firms in the U.S. regarding their foreign exchange risk management. Most respondents in his survey strongly object to the hedging strategies as suggested by expected utility maximization because they regard those strategies as ex-post suboptimal ones, which is consistent with regret theory.

The purpose of this paper is to incorporate regret theory into the model of the multinational firm under exchange rate uncertainty à la Broll and Zilcha (1992). To this end, we characterize the multinational firm's regret-averse preferences by a modified utility function that includes disutility from having chosen ex-post suboptimal alternatives. The extent of regret depends on the difference between the actual home currency profit and the maximum home currency profit attained by making the optimal production and export decisions had the multinational firm observed the true realization of the random spot exchange rate. ${ }^{1} \mathrm{We}$ are particularly interested in examining the impact of regret on the multinational firm's production and export decisions as compared to the benchmark case of certainty.

We show that the multinational firm optimally produces less, sells more domestically, and exports less abroad under uncertainty than under certainty should the multinational firm be not too regret averse. In this case, the risk-sharing motive remains first-order important to the multinational firm. These findings suggest that it is quite possible that the multinational firm may optimally produce more, sell less domestically, and export more abroad under uncertainty than under certainty should the multinational firm be sufficiently regret averse. To verify this conjecture, we develop a binary model wherein the random spot

\footnotetext{
${ }^{1}$ In our model, regret is driven by the lack of flexibility that the multinational firm can react to new information, which is in stark contrast to the real options approach.
} 
exchange rate can take on either a low value or a high value with positive probability. In such a binary framework, we show that the conventional results are violated if the multinational firm is sufficiently regret averse and the low spot exchange rate is very likely to prevail. In this case, the optimal levels of domestic sales and foreign exports under certainty are very close to their counterparts that are ex-post optimal at the low spot exchange rate. The sufficiently regret-averse multinational firm as such optimally adjusts its level of foreign exports upward and its level of domestic sales downward so as to limit the potential regret when the high spot exchange rate is actually revealed, thereby rendering the optimal output level under uncertainty to exceed that under certainty.

Our novel results that regret aversion may lead to more foreign exports and less domestic sales are likely to hold in a country having a pegged exchange rate with a small probability that the country has to devalue its currency. In case of the unlikely event of devaluation, multinational firms earn more on their foreign exports in domestic currency terms and thus would have regret from the fact that they did not sell enough abroad. This suggests that multinational firms in countries with pegged exchange rates tend to export more, other things being equal. Indeed, Klein and Shambaugh (2006) find that pegging appears to increase trade by up to $35 \%$, whereas indirect pegs do not appear to have any strong impact on trade.

This paper is closely related to the early work of Paroush and Venezia (1979) who examine Sandmo's (1971) model of the competitive firm under uncertainty wherein the firm possesses a bivariate utility function defined on profits and regret. Our modified regret-theoretical utility function is a tractable version of theirs in that the bivariate utility function is specified as an additive separable function such that the degree of regret can be measured by a constant coefficient. ${ }^{2}$ In contrast to Paroush and Venezia (1979), we consider Broll and Zilcha's (1992) model of the multinational firm that possesses market power and sells in the home and foreign countries. ${ }^{3}$

\footnotetext{
${ }^{2}$ See Wong (2014) for an application of the modified regret-theoretical utility function in the context of Paroush and Venezia (1979).

${ }^{3}$ Our results also apply when the firm cannot sell domestically and has to export to the foreign country
} 
The rest of this paper is organized as follows. Section 2 delineates the model of the multinational firm under exchange rate uncertainty (Broll and Zilcha 1992). The multinational firm's preferences exhibit not only risk aversion but also regret aversion. Section 3 solves the model and provides sufficient conditions under which the behavior of the regret-averse multinational firm is qualitatively the same as that of the risk-averse multinational firm. Section 4 develops a binary model to show the possibility that introducing regret aversion to the multinational firm may induce the multinational firm to optimally produce more, sell less domestically, and export more abroad under uncertainty than under certainty. The final section concludes.

\section{The Model}

Consider the multinational firm under exchange rate uncertainty $\grave{a}$ la Broll and Zilcha (1992). There is one period with two dates, 0 and 1 . To begin, the multinational firm produces a single commodity in the home country according to a deterministic cost function, $C(Q)$, where $Q \geq 0$ is the output level, and $C(Q)$ is compounded to date 1 with the properties that $C(0)=C^{\prime}(0)=0$, and $C^{\prime}(Q)>0$ and $C^{\prime \prime}(Q)>0$ for all $Q>0 .{ }^{4}$ The multinational firm commits to selling $Q_{1}$ units of its output at home and exporting $Q_{2}$ units to a foreign country, where $Q_{1} \geq 0, Q_{2} \geq 0$, and $Q_{1}+Q_{2}=Q$.

The multinational firm's domestic sales generate home currency revenues at date 1 specified by a deterministic revenue function, $R_{1}\left(Q_{1}\right)$, where $R_{1}(0)=0$, and $R_{1}^{\prime}\left(Q_{1}\right)>0$ and $R_{1}^{\prime \prime}\left(Q_{1}\right)<0$ for all $Q_{1} \geq 0$. On the other hand, the multinational firm's exports generate foreign currency receives at date 1 specified by another deterministic revenue function, $R_{2}\left(Q_{2}\right)$, where $R_{2}(0)=0$, and $R_{2}^{\prime}\left(Q_{1}\right)>0$ and $R_{2}^{\prime \prime}\left(Q_{2}\right)<0$ for all $Q_{2} \geq 0$.

only, i.e., the firm is an exporting firm.

${ }^{4}$ The strict convexity of the cost function reflects the fact that the multinational firm's production technology exhibits decreasing returns to scale. Our qualitative results remain intact if the multinational firm can produce the commodity in both the home and foreign countries according to the respective deterministic cost functions. 
Due to the segmentation of the home and foreign markets, arbitrage transactions are either impossible or unprofitable, thereby invalidating the law of one price. ${ }^{5}$

We model the exchange rate uncertainty by a random variable, $\tilde{S}$, that denotes the spot exchange rate at date 1 and is expressed in units of the home currency per unit of the foreign currency. ${ }^{6} \quad \tilde{S}$ is distributed according to a known cumulative distribution function, $F(S)$, over support $[\underline{S}, \bar{S}]$, where $0<\underline{S}<\bar{S} .^{7}$ The multinational firm's home currency profit at date 1 is given by

$$
\Pi(\tilde{S})=R_{1}\left(Q_{1}\right)+\tilde{S} R_{2}\left(Q_{2}\right)-C\left(Q_{1}+Q_{2}\right)
$$

According to Paroush and Venezia (1979), the multinational firm is regret-averse if its preferences are represented by a bivariate utility function, $V(\Pi, D)$, defined on profits and regret, where $\Pi \geq 0$ is the multinational firm's home currency profit at date 1 , and $D=\Pi^{\max }-\Pi \geq 0$ is the regret that is equal to the difference between the actual home currency profit at date $1, \Pi$, and the maximum home currency profit, $\Pi^{\text {max }}$, that the multinational firm could have earned at date 1 if multinational firm had made the optimal production and export decisions based on knowing the realized spot exchange rate. Paroush and Venezia (1979) assume that $V_{\Pi}(\Pi, D)>0, V_{D}(\Pi, D)<0, V_{\Pi \Pi}(\Pi, D)<0, V_{D D}(\Pi, D)<$ $0, V_{\Pi \Pi}(\Pi, D) V_{D D}(\Pi, D)>V_{\Pi D}(\Pi, D)^{2}$, and $V_{\Pi \Pi}(\Pi, D)+V_{D D}(\Pi, D)-2 V_{\Pi D}(\Pi, D)<0$, where subscripts denote partial derivatives.

For tractability, we adopt the following specification of $V(\Pi, D)$ as proposed by Braun and Muermann (2004) and Wong (2011, 2012):

$$
V(\Pi, D)=U(\Pi)-\beta G(D),
$$

where $U(\Pi)$ is a von Neumann-Morgenstern utility function with $U^{\prime}(\Pi)>0$ and $U^{\prime \prime}(\Pi)<0$,

\footnotetext{
${ }^{5}$ Engel and Rogers $(1996,2001)$ and Parsley and Wei (1996) provide supportive evidence that arbitrage transactions among national markets are indeed imperfect.

${ }^{6}$ Throughout the paper, random variables have a tilde $(\sim)$ while their realizations do not.

${ }^{7}$ An alternative way to model the exchange rate uncertainty is to apply the concept of information systems that are conditional cumulative distribution functions over a set of signals imperfectly correlated with $\tilde{S}$ (Broll et al., 2013).
} 
$\beta>0$ is a constant regret coefficient, and $G(D)$ is a regret function such that $G(0)=0$, and $G^{\prime}(D)>0$ and $G^{\prime \prime}(D)>0$ for all $D \geq 0 .{ }^{8}$ It is easily verified that all the assumptions made by Paroush and Venezia (1979) are satisfied by the additive separable utility function given by Eq. (2). Since $\Pi$ cannot exceed $\Pi^{\text {max }}$, the multinational firm experiences disutility from forgoing the possibility of undertaking the ex-post optimal production and export decisions.

To characterize the regret-averse multinational firm's optimal production and export decisions, we have to first determine the maximum home currency profit, $\Pi^{\max }$, if the multinational firm could have observed the realized spot exchange rate, $S$. In this case, the optimal levels of domestic sales and foreign exports are given by $Q_{1}(S)$ and $Q_{2}(S)$, respectively, which are the solution to the following system of equations:

$$
R_{1}^{\prime}\left[Q_{1}(S)\right]=C^{\prime}\left[Q_{1}(S)+Q_{2}(S)\right]
$$

and

$$
S R_{2}^{\prime}\left[Q_{2}(S)\right]=C^{\prime}\left[Q_{1}(S)+Q_{2}(S)\right]
$$

Differentiating Eqs. (3) and (4) with respect to $S$ and rearranging terms yields

$$
Q_{1}^{\prime}(S)=-\frac{R_{2}^{\prime}\left[Q_{2}(S)\right] C^{\prime \prime}\left[Q_{1}(S)+Q_{2}(S)\right]}{S R_{1}^{\prime \prime}\left[Q_{1}(S)\right] R_{2}^{\prime \prime}\left[Q_{2}(S)\right]-\left\{R_{1}^{\prime \prime}\left[Q_{1}(S)\right]+S R_{2}^{\prime \prime}\left[Q_{2}(S)\right]\right\} C^{\prime \prime}[Q(S)]}<0
$$

and

$$
Q_{2}^{\prime}(S)=\frac{R_{2}^{\prime}\left[Q_{2}(S)\right]\left\{C^{\prime \prime}[Q(S)]-R_{1}^{\prime \prime}\left[Q_{1}(S)\right]\right\}}{S R_{1}^{\prime \prime}\left[Q_{1}(S)\right] R_{2}^{\prime \prime}\left[Q_{2}(S)\right]-\left\{R_{1}^{\prime \prime}\left[Q_{1}(S)\right]+S R_{2}^{\prime \prime}\left[Q_{2}(S)\right]\right\} C^{\prime \prime}[Q(S)]}>0
$$

where $Q(S)=Q_{1}(S)+Q_{2}(S)$. The maximum home currency profit at date 1 as a function of $S$ is then given by

$$
\Pi^{\max }(S)=R_{1}\left[Q_{1}(S)\right]+S R_{2}\left[Q_{2}(S)\right]-C\left[Q_{1}(S)+Q_{2}(S)\right]
$$

\footnotetext{
${ }^{8}$ Braun, and Muermann (2004) and Wong (2011, 2012) consider a regret function that depends on the difference between the utility level of the actual home currency profit and that of the maximum home currency profit, $U\left(\Pi^{\max }\right)-U(\Pi)$. Since such a specification is simply a monotonic transformation of ours, none of the qualitative results are affected if we adopt this alternative approach (see also Wong 2014).
} 
Differentiating Eq. (7) with respect to $S$ and using Eqs. (3) and (4) yields $\Pi^{\max ^{\prime}}(S)=$ $R_{2}\left[Q_{2}(S)\right]>0$.

We can now state the regret-averse multinational firm's ex-ante decision problem. At date 0 , the multinational firm chooses the levels of domestic sales and foreign exports, $Q_{1}$ and $Q_{2}$, so as to maximize the expected value of its regret-theoretical utility function:

$$
\max _{Q_{1} \geq 0, Q_{2} \geq 0} \mathrm{E}\left\{U[\Pi(\tilde{S})]-\beta G\left[\Pi^{\max }(\tilde{S})-\Pi(\tilde{S})\right]\right\}
$$

where $\Pi(\tilde{S})$ and $\Pi^{\max }(\tilde{S})$ are given by Eqs. (1) and (7), respectively, and $\mathrm{E}(\cdot)$ is the expectation operator with respect to the cumulative distribution function, $F(S)$. The firstorder conditions for program (8) are given by

$$
R_{1}^{\prime}\left(Q_{1}^{*}\right)-C^{\prime}\left(Q_{1}^{*}+Q_{2}^{*}\right)=0
$$

and

$$
\mathrm{E}\left\{\left\{U^{\prime}\left[\Pi^{*}(\tilde{S})\right]+\beta G^{\prime}\left[\Pi^{\max }(\tilde{S})-\Pi^{*}(\tilde{S})\right]\right\}\left[\tilde{S} R_{2}^{\prime}\left(Q_{2}^{*}\right)-C^{\prime}\left(Q_{1}^{*}+Q_{2}^{*}\right)\right]\right\}=0
$$

where an asterisk $\left(^{*}\right)$ indicates an optimal level. The second-order conditions for program (8) are satisfied given the assumed properties of $U(\Pi), G(D), R_{1}\left(Q_{1}\right), R_{2}\left(Q_{2}\right)$, and $C(Q)$.

\section{Impact of Regret on Production and Export Decisions}

As a benchmark, we suppose that the uncertain spot exchange rate, $\tilde{S}$, is fixed at its expected value, $\mathrm{E}(\tilde{S})$. In this benchmark case of certainty, Eqs. (9) and (10) reduce to

$$
R_{1}^{\prime}\left(Q_{1}^{\circ}\right)-C^{\prime}\left(Q_{1}^{\circ}+Q_{2}^{\circ}\right)=0
$$

and

$$
\mathrm{E}(\tilde{S}) R_{2}^{\prime}\left(Q_{2}^{\circ}\right)-C^{\prime}\left(Q_{1}^{\circ}+Q_{2}^{\circ}\right)=0
$$


where $Q_{1}^{\circ}$ and $Q_{2}^{\circ}$ are the optimal levels of domestic sales and foreign exports, respectively. We are interested in comparing $Q_{1}^{*}$ with $Q_{1}^{\circ}$ and $Q_{2}^{*}$ with $Q_{2}^{\circ}$.

Let $Q_{1}\left(Q_{2}\right)$ be the solution to $R_{1}^{\prime}\left[Q_{1}\left(Q_{2}\right)\right]=C^{\prime}\left[Q_{1}\left(Q_{2}\right)+Q_{2}\right]$. Then, Eqs. (9) and (11) imply that $Q_{1}\left(Q_{2}^{*}\right)=Q_{1}^{*}$ and $Q_{1}\left(Q_{2}^{\circ}\right)=Q_{1}^{\circ}$, respectively. Furthermore, we have

$$
Q_{1}^{\prime}\left(Q_{2}\right)=\frac{C^{\prime \prime}\left[Q_{1}\left(Q_{2}\right)+Q_{2}\right]}{R_{1}^{\prime \prime}\left[Q_{1}\left(Q_{2}\right)\right]-C^{\prime \prime}\left[Q_{1}\left(Q_{2}\right)+Q_{2}\right]}<0
$$

since $C^{\prime \prime}(Q)>0$ and $R_{1}^{\prime \prime}\left(Q_{1}\right)<0$. Substituting $Q_{1}\left(Q_{2}\right)$ into the objective function of program (8) and differentiating with respect to $Q_{2}$ yields

$$
\begin{aligned}
& \left.\frac{\partial \mathrm{E}\left\{V\left[\Pi(\tilde{S}), \Pi^{\max }(\tilde{S})-\Pi(\tilde{S})\right]\right\}}{\partial Q_{2}}\right|_{Q_{1}=Q_{1}\left(Q_{2}\right)} \\
& =\mathrm{E}\left\{\left\{U^{\prime}[\hat{\Pi}(\tilde{S})]+\beta G^{\prime}\left[\Pi^{\max }(\tilde{S})-\hat{\Pi}(\tilde{S})\right]\right\}\left\{\tilde{S} R_{2}^{\prime}\left(Q_{2}\right)-C^{\prime}\left[Q_{1}\left(Q_{2}\right)+Q_{2}\right]\right\}\right\},
\end{aligned}
$$

where $\hat{\Pi}(S)=R_{1}\left[Q_{1}\left(Q_{2}\right)\right]+S R_{2}\left(Q_{2}\right)-C\left[Q_{1}\left(Q_{2}\right)+Q_{2}\right]$. Evaluating Eq. (14) at $Q_{2}=Q_{2}^{\circ}$ yields

$$
\left.\frac{\partial \mathrm{E}\left\{V\left[\Pi(\tilde{S}), \Pi^{\max }(\tilde{S})-\Pi(\tilde{S})\right]\right\}}{\partial Q_{2}}\right|_{Q_{1}=Q_{1}^{\circ}, Q_{2}=Q_{2}^{\circ}}=\mathrm{E}\{\Phi(\tilde{S})[\tilde{S}-\mathrm{E}(\tilde{S})]\} R_{2}^{\prime}\left(Q_{2}^{\circ}\right)
$$

where $\Phi(\tilde{S})=U^{\prime}\left[\Pi^{\circ}(\tilde{S})\right]+\beta G^{\prime}\left[\Pi^{\max }(\tilde{S})-\Pi^{\circ}(\tilde{S})\right]$, and we have used Eq. (12) and $Q_{1}\left(Q_{2}^{\circ}\right)=$ $Q_{1}^{\circ}$. If the right-hand side of Eq. (15) is negative (positive), it follows from Eq. (9) and the second-order conditions for program (8) that $Q_{2}^{*}<(>) Q_{2}^{\circ}$. Eq. (13) then implies that $Q_{1}^{*}>(<) Q_{1}^{\circ}$. Since $R_{1}^{\prime \prime}\left(Q_{1}\right)<0$ and $C^{\prime \prime}(Q)>0$, it follows from Eqs. (9) and (11) that $Q_{1}^{*}+Q_{2}^{*}<(>) Q_{1}^{\circ}+Q_{2}^{\circ}$.

The following proposition provides sufficient conditions under which the right-hand side of Eq. (15) is unambiguously negative.

Proposition 1 If $U^{\prime \prime \prime}(\Pi) \geq 0$ and $G^{\prime \prime \prime}(D) \geq 0$, then a sufficient condition that ensures the regret-averse multinational firm to produce less, i.e., $Q^{*}<Q^{\circ}$, sells more in the home market, i.e., $Q_{1}^{*}>Q_{2}^{\circ}$, and exports less to the foreign country, i.e., $Q_{2}^{*}<Q_{2}^{\circ}$, as compared 
to the optimal levels under certainty, is that the constant regret coefficient, $\beta$, is sufficiently small such that

$$
\beta \leq \frac{U^{\prime}\left\{\Pi^{\circ}[\mathrm{E}(\tilde{S})]\right\}-U^{\prime}\left[\Pi^{\circ}(\bar{S})\right]}{G^{\prime}\left[\Pi^{\max }(\bar{S})-\Pi^{\circ}(\bar{S})\right]-G^{\prime}(0)}
$$

where $\Pi^{\circ}(S)=R_{1}\left(Q_{1}^{\circ}\right)+S R_{2}\left(Q_{2}^{\circ}\right)-C\left(Q_{1}^{\circ}+Q_{2}^{\circ}\right)$.

Proof We can write Eq. (15) as

$$
\left.\frac{\partial \mathrm{E}\{V[\Pi(\tilde{S})]\}}{\partial Q_{2}}\right|_{Q_{1}=Q_{1}^{\circ}, Q_{2}=Q_{2}^{\circ}}=\mathrm{E}\{\{\Phi(\tilde{S})-\Phi[\mathrm{E}(\tilde{S})]\}[\tilde{S}-\mathrm{E}(\tilde{S})]\} R_{2}^{\prime}\left(Q_{2}^{\circ}\right)
$$

We want to show that $\Phi(S)>(<) \Phi[\mathrm{E}(\tilde{S})]$ for all $S<(>) \mathrm{E}(\tilde{S})$ so that the right-hand side of Eq. (17) is negative. Eq. (9) and the second-order conditions for program (8) as such imply that $Q_{2}^{*}<Q_{2}^{\circ}$. It then follows from Eq. (13) that $Q_{1}^{*}>Q_{1}^{\circ}$. Since $R_{1}^{\prime \prime}\left(Q_{1}\right)<0$ and $C^{\prime \prime}(Q)>0$, Eqs. (9) and (11) imply that $Q_{1}^{*}+Q_{2}^{*}<Q_{1}^{\circ}+Q_{2}^{\circ}$.

Differentiating $\Phi(S)$ twice with respect to $S$ yields

$$
\Phi^{\prime}(S)=U^{\prime \prime}\left[\Pi^{\circ}(S)\right] R_{2}\left(Q_{2}^{\circ}\right)+\beta G^{\prime \prime}\left[\Pi^{\max }(S)-\Pi^{\circ}(S)\right]\left\{R_{2}\left[Q_{2}(S)\right]-R_{2}\left(Q_{2}^{\circ}\right)\right\}
$$

and

$$
\begin{aligned}
\Phi^{\prime \prime}(S)= & U^{\prime \prime \prime}\left[\Pi^{\circ}(S)\right] R_{2}\left(Q_{2}^{\circ}\right)^{2}+\beta G^{\prime \prime \prime}\left[\Pi^{\max }(S)-\Pi^{\circ}(S)\right]\left\{R_{2}\left[Q_{2}(S)\right]-R_{2}\left(Q_{2}^{\circ}\right)\right\}^{2} \\
& +\beta G^{\prime \prime}\left[\Pi^{\max }(S)-\Pi^{\circ}(S)\right] R_{2}^{\prime}\left[Q_{2}(S)\right] Q_{2}^{\prime}(S) .
\end{aligned}
$$

Since $U^{\prime \prime \prime}(\Pi) \geq 0$ and $G^{\prime \prime \prime}(D) \geq 0$, it follows from Eq. (19) that $\Phi^{\prime \prime}(S)>0$ for all $S \in[\underline{S}, \bar{S}]$. Eq. (6) implies that $Q_{2}(S)<(>) Q_{2}^{\circ}$ for all $S<(>) \mathrm{E}(\tilde{S})$. It then follows from Eq. (18) that $\Phi^{\prime}(S)<0$ for all $S \leq \mathrm{E}(\tilde{S})$ and thus $\Phi(S)>\Phi[\mathrm{E}(\tilde{S})]$ for all $S<\mathrm{E}(\tilde{S})$. Note that

$$
\Phi(\bar{S})-\Phi[\mathrm{E}(\tilde{S})]=U^{\prime}\left[\Pi^{\circ}(\bar{S})\right]+\beta G^{\prime}\left[\Pi^{\max }(\bar{S})-\Pi^{\circ}(\bar{S})\right]-U^{\prime}\left\{\Pi^{\circ}[\mathrm{E}(\tilde{S})]\right\}-\beta G^{\prime}(0)
$$


It follows from Eq. (20) and condition (16) that $\Phi[\mathrm{E}(\tilde{S})] \geq \Phi(\bar{S})$. Since $\Pi^{\circ}[\mathrm{E}(\tilde{S})]<\Pi^{\circ}(\bar{S})$ and $U^{\prime \prime}(\Pi)<0$, we have $U^{\prime}\left\{\Pi^{\circ}[\mathrm{E}(\tilde{S})]\right\}>U^{\prime}\left[\Pi^{\circ}(\bar{S})\right]$. Furthermore, $\Pi^{\max }(\bar{S})>\Pi^{\circ}(\bar{S})$ and $G^{\prime \prime}(D)>0$ so that $G^{\prime}\left[\Pi^{\max }(\bar{S})-\Pi^{\circ}(\bar{S})\right]>G^{\prime}(0)$. The right-hand side of condition (16) as such is strictly positive so that condition (16) is non-trivial. Since $\Phi(S)$ is strictly convex in $S$ and $\Phi^{\prime}[\mathrm{E}(\tilde{S})]<0$, it follows from condition $(16)$ that $\Phi(S)<\Phi[\mathrm{E}(\tilde{S})]$ for all $S>\mathrm{E}(\tilde{S})$. This completes our proof.

The intuition for Proposition 1 is as follows. If $\beta=0$, the multinational firm is purely risk averse. Broll and Zilcha (1992) show that the risk-averse multinational firm produces less than $Q^{\circ}$, sells more than $Q_{1}^{\circ}$ domestically, and exports less than $Q_{2}^{\circ}$ to the foreign country so as to limit its exposure to the exchange rate uncertainty. For $\beta$ sufficiently small, introducing regret aversion to the multinational firm would not substantially change such a risk-sharing motive, thereby rendering $Q^{*}<Q^{\circ}, Q_{1}^{*}>Q_{1}^{\circ}$, and $Q_{2}^{*}<Q_{2}^{\circ}$.

\section{A Binary Model}

To gain more insights, we consider in this section a simple binary model such that the random spot exchange rate, $\tilde{S}$, takes on the low value, $\underline{S}$, with probability $p$ and the high value, $\bar{S}$, with probability $1-p$, where $0<p<1$. In such a binary model, the right-hand side of Eq. (15) becomes

$$
\begin{aligned}
& p\left\{U^{\prime}\left[\Pi^{\circ}(\underline{S})\right]+\beta G^{\prime}\left[\Pi^{\max }(\underline{S})-\Pi^{\circ}(\underline{S})\right]\right\}[\underline{S}-p \underline{S}-(1-p) \bar{S}] R_{2}^{\prime}\left(Q_{2}^{\circ}\right) \\
& +(1-p)\left\{U^{\prime}\left[\Pi^{\circ}(\bar{S})\right]+\beta G^{\prime}\left[\Pi^{\max }(\bar{S})-\Pi^{\circ}(\bar{S})\right]\right\}[\bar{S}-p \underline{S}-(1-p) \bar{S}] R_{2}^{\prime}\left(Q_{2}^{\circ}\right) \\
& =p(1-p)(\bar{S}-\underline{S}) R_{2}^{\prime}\left(Q_{2}^{\circ}\right) \Psi(p),
\end{aligned}
$$

where $\Psi(p)=U^{\prime}\left[\Pi^{\circ}(\bar{S})\right]+\beta G^{\prime}\left[\Pi^{\max }(\bar{S})-\Pi^{\circ}(\bar{S})\right]-U^{\prime}\left[\Pi^{\circ}(\underline{S})\right]-\beta G^{\prime}\left[\Pi^{\max }(\underline{S})-\Pi^{\circ}(\underline{S})\right]$. If right-hand side of Eq. (21) is negative (positive), i.e., $\Psi(p)<(>) 0$, it follows from Eq. (9) 
and the second-order conditions for program (8) that $Q_{2}^{*}<(>) Q_{2}^{\circ}$. Eq. (13) then implies that $Q_{1}^{*}>(<) Q_{1}^{\circ}$. Since $R_{1}^{\prime \prime}\left(Q_{1}\right)<0$ and $C^{\prime \prime}(Q)>0$, it follows from Eqs. (9) and (11) that $Q_{1}^{*}+Q_{2}^{*}<(>) Q_{1}^{\circ}+Q_{2}^{\circ}$.

We state and prove the following proposition.

Proposition 2 Suppose that the random spot exchange rate, $\tilde{S}$, can take on the low value, $\underline{S}$, with probability $p$ and the high value, $\bar{S}$, with probability $1-p$, where $0<p<1$. If the constant regret coefficient, $\beta$, is sufficiently small such that

$$
\beta \leq \frac{U^{\prime}\left[\Pi^{\max }(\underline{S})\right]-U^{\prime}\left\{R_{1}\left[Q_{1}(\underline{S})\right]+\bar{S} R_{2}\left[Q_{2}(\underline{S})\right]-C[Q(\underline{S})]\right\}}{G^{\prime}\left\{\Pi^{\max }(\bar{S})-R_{1}\left[Q_{1}(\underline{S})\right]-\bar{S} R_{2}\left[Q_{2}(\underline{S})\right]+C[Q(\underline{S})]\right\}-G^{\prime}(0)}
$$

the regret-averse multinational firm optimally produces less, i.e., $Q^{*}<Q^{\circ}$, sells more in the home market, i.e., $Q_{1}^{*}>Q_{1}^{\circ}$, and exports less to the foreign country, i.e., $Q_{2}^{*}<Q_{2}^{\circ}$, as compared to the optimal levels under certainty. If $\beta$ is sufficiently large such that condition (22) does not hold, there exists a unique value, $p^{*} \in(0,1)$, that solves $\Psi\left(p^{*}\right)=0$, such that $Q^{*}<(>) Q^{\circ}, Q_{1}^{*}>(<) Q_{1}^{\circ}$, and $Q_{2}^{*}<(>) Q_{2}^{\circ}$, for all $p<(>) p^{*}$.

Proof In the binary model, we can write Eq. (12) as

$$
[p \underline{S}+(1-p) \bar{S}] R_{2}^{\prime}\left(Q_{2}^{\circ}\right)-C^{\prime}\left[Q_{1}\left(Q_{2}^{\circ}\right)+Q_{2}^{\circ}\right]=0
$$

since $Q_{1}\left(Q_{2}^{\circ}\right)=Q_{1}^{\circ}$. Differentiate Eq. (23) with respect to $p$ yields

$$
\frac{\mathrm{d} Q_{2}^{\circ}}{\mathrm{d} p}=\frac{(\bar{S}-\underline{S}) R_{2}^{\prime}\left(Q_{2}^{\circ}\right)}{[p \underline{S}+(1-p) \bar{S}] R_{2}^{\prime \prime}\left(Q_{2}^{\circ}\right)-C^{\prime \prime}\left[Q_{1}\left(Q_{2}^{\circ}\right)+Q_{2}^{\circ}\right]\left[Q_{1}^{\prime}\left(Q_{2}^{\circ}\right)+1\right]}<0,
$$

since $R_{2}^{\prime \prime}\left(Q_{2}\right)<0, C^{\prime \prime}(Q)>0$, and Eq. (13) implies that $Q_{1}^{\prime}\left(Q_{2}\right)+1>0$. Differentiating $\Psi(p)$ with respect to $p$ yields

$$
\begin{aligned}
\Psi^{\prime}(p)= & (\bar{S}-\underline{S}) R_{2}^{\prime}\left(Q_{2}^{\circ}\right) \frac{\mathrm{d} Q_{2}^{\circ}}{\mathrm{d} p}\left\{p U^{\prime \prime}\left[\Pi^{\circ}(\bar{S})\right]-p \beta G^{\prime \prime}\left[\Pi^{\max }(\bar{S})-\Pi^{\circ}(\bar{S})\right]\right. \\
& \left.+(1-p) U^{\prime \prime}\left[\Pi^{\circ}(\underline{S})\right]-(1-p) \beta G^{\prime \prime}\left[\Pi^{\max }(\underline{S})-\Pi^{\circ}(\underline{S})\right]\right\}>0
\end{aligned}
$$


where we have used Eqs. (11) and (23), and the inequality follows from $U^{\prime \prime}(\Pi)<0$, $G^{\prime \prime}(D)>0$, and Eq. (24). At $p=0$, we have $Q_{1}^{\circ}=Q_{1}(\bar{S})$ and $Q_{2}^{\circ}=Q_{2}(\bar{S})$. In this case, $\Pi^{\circ}(\bar{S})=\Pi^{\max }(\bar{S})>\Pi^{\max }(\underline{S})>\Pi^{\circ}(\underline{S})$. Hence, we have

$$
\Psi(0)=U^{\prime}\left[\Pi^{\max }(\bar{S})\right]-U^{\prime}\left[\Pi^{\circ}(\underline{S})\right]+\beta\left\{G^{\prime}(0)-G^{\prime}\left[\Pi^{\max }(\underline{S})-\Pi^{\circ}(\underline{S})\right]\right\}<0,
$$

since $U^{\prime \prime}(\Pi)<0, G^{\prime \prime}(D)>0$, and $\Pi^{\max }(\bar{S})>\Pi^{\max }(\underline{S})$. On the other hand, at $p=1$, we have $Q_{1}^{\circ}=Q_{1}(\underline{S})$ and $Q_{2}^{\circ}=Q_{2}(\underline{S})$. In this case, $\Pi^{\circ}(\underline{S})=\Pi^{\max }(\underline{S})<\Pi^{\circ}(\bar{S})<\Pi^{\max }(\bar{S})$. Hence, we have

$$
\Psi(1)=U^{\prime}\left[\Pi^{\circ}(\bar{S})\right]-U^{\prime}\left[\Pi^{\max }(\underline{S})\right]+\beta\left\{G^{\prime}\left[\Pi^{\max }(\bar{S})-\Pi^{\circ}(\bar{S})\right]-G^{\prime}(0)\right\}
$$

It follows from Eq. (27) and condition $(22)$ that $\Psi(1) \leq 0$. In this case, Eq. (25) implies that $\Psi(p)<0$ for all $p \in(0,1)$ so that $Q_{2}^{*}<Q_{2}^{\circ}$. Eq. (13) then implies that $Q_{1}^{*}>Q_{1}^{\circ}$. Since $R_{1}^{\prime \prime}\left(Q_{1}\right)<0$ and $C^{\prime \prime}(Q)>0$, it follows from Eqs. (9) and (11) that $Q_{1}^{*}+Q_{2}^{*}<Q_{1}^{\circ}+Q_{2}^{\circ}$.

If condition (22) does not hold, we have $\Psi(1)>0$. It then follows from Eq. (25) that there exists a unique point, $p^{*} \in(0,1)$, such that $\Psi(p)<(>) 0$ for all $p<(>) p^{*}$. Hence, in this case, we have $Q_{2}^{*}<(>) Q_{2}^{\circ}$, thereby $Q_{1}^{*}>(<) Q_{1}^{\circ}$ and $Q_{1}^{*}+Q_{2}^{*}<(>) Q_{1}^{\circ}+Q_{2}^{\circ}$, for all $p<(>) p^{*}$.

The intuition for Proposition 2 is as follows. If condition (22) holds, the constant regret coefficient, $\beta$, is sufficiently small so that the risk-sharing motive remains the dominant factor in determining the multinational firm's production and export decisions. Hence, we have $Q^{*}<Q^{\circ}, Q_{1}^{*}>Q_{1}^{\circ}$, and $Q_{2}^{*}<Q_{2}^{\circ}$, which are consistent with the results of Proposition 1. If condition (22) does not hold, $\beta$ is sufficiently large so that both the risk-sharing and regret-aversion motives have the first-order effect on the multinational firm's behavior. When $\bar{S}$ is very likely to be seen at date $1, Q_{2}^{\circ}$ is closer to $Q_{2}(\bar{S})$ and further way from $Q_{2}(\underline{S})$. Introducing regret aversion, which is sufficiently severe, to the multinational firm makes the firm take into account the substantial disutility from the large discrepancy of its export level, $Q_{2}^{\circ}-Q_{2}(\underline{S})$, when the low spot exchange rate is revealed. To avoid regret, 
the regret-averse multinational firm optimally adjusts its export level downward from $Q_{2}^{\circ}$ to move closer to $Q_{2}(\underline{S})$ when $p$ is small. In this case, the regret-aversion motive reinforces the risk-sharing motive so that $Q_{1}^{*}>Q_{1}^{\circ}, Q_{2}^{*}<Q_{2}^{\circ}$, and $Q^{*}<Q^{\circ}$. On the other hand, when $\underline{S}$ is very likely to be seen at date $1, Q_{2}^{\circ}$ is close to $Q_{2}(\underline{S})$ and further way from $Q_{2}(\bar{S})$. The regret-averse multinational firm as such optimally adjusts its export level upward from $Q_{2}^{\circ}$ to reduce the discrepancy of its export level, $Q_{2}(\bar{S})-Q_{2}^{*}$, when the high spot exchange rate is revealed. In this case, the regret-aversion motive counteracts the risk-sharing motive when $p$ is large. Hence, there exists a critical value, $p^{*}$, such that $Q^{*}<(>) Q^{\circ}, Q_{1}^{*}>(<) Q_{1}^{\circ}$, and $Q_{2}^{*}<(>) Q_{2}^{\circ}$ whenever $p<(>) p^{*}$.

\section{Conclusion}

In this paper, we incorporate regret theory into Broll and Zilcha's (1982) model of the multinational firm under exchange rate uncertainty. The multinational firm simultaneously sells in the home market and exports to a foreign country. We characterize the multinational firm's regret-averse preferences by a modified utility function that includes disutility from having chosen ex-post suboptimal alternatives. The extent of regret depends on the difference between the actual home currency profit and the maximum home currency profit attained by making the optimal production and export decisions had the multinational firm observed the true realization of the random spot exchange rate.

We show that the conventional results of the extant literature that the multinational firm optimally produces less, sells more domestically, and exports less abroad when the exchange rate uncertainty prevails than when the random spot exchange rate is fixed at the expected value should the multinational firm be not too regret averse. These findings suggest that it is possible that the multinational firm may optimally produces more, sells less domestically, and exports more abroad under uncertainty than under certainty. We verify such a conjecture by using a simple binary model wherein the random spot exchange 
rate can take on either a low value or a high value with positive probability. We show that the non-conventional results hold in the binary model if the multinational firm is sufficiently regret averse and the low spot exchange rate is very likely to prevail. Regret aversion as such plays a distinctive role, vis-à-vis risk aversion, in shaping the production and export decisions of the multinational firm under exchange rate uncertainty.

\section{References}

Bell DE (1982) Regret in decision making under uncertainty. Operations Research 30:961981

Bell DE (1983) Risk premiums for decision regret. Management Science 29:1156-1166

Braun M, Muermann A. (2004) The impact of regret on the demand for insurance. Journal of Risk and Insurance 71:737-767

Broll U, Eckwert B, Wong KP (2014). Transparency and risk sharing in international trade. The Manchester School, in press

Broll U, Welzel P, Wong KP (2009) Export and strategic currency hedging. Open Economies Review 20:717-732

Broll U, Wong KP (2006) Multinationals, hedging, and capital structure under exchange rate uncertainty. Open Economies Review 17:103-114

Broll U, Zilcha I (1992) Exchange rate uncertainty, futures markets and the multinational firm. European Economic Review 36:815-826

Engel C, Rogers JH (1996) How wide is the border? American Economic Review 86:11121125

Engel C, Rogers JH (2001) Violating the law of one price: should we make a Federal case out of it? Journal of Money, Credit and Banking 33:1-15 
Fillat JL, Garetto S (2010) Risk, returns, and multinational production. Working paper, Department of Economics, Boston University

Klein MW, Shambaugh JC (2006) Fixed exchange rate and trade. Journal of International Economics 70:359-383

Loomes G (1988) Further evidence of the impact of regret and disappointment in choice under uncertainty. Economica 55:47-62

Loomes G, Starmer C, Sugden R (1992) Are preferences monotonic - testing some predictions of regret theory. Economica 59:17-33

Loomes G, Sugden R (1982) Regret theory: an alternative theory of rational choice under uncertainty. Economic Journal 92:805-824

Loomes G, Sugden R (1987) Testing for regret and disappointment in choice under uncertainty. Economic Journal 97:118-129

Mrázová M, Neary JP (2011) Selection effects with heterogeneous firms. Discussion paper no. 588, Department of Economics, University of Oxford

Paroush J, Venezia I (1979) On the theory of the competitive firm with a utility function defined on profits and regret. European Economic Review 12:193-202.

Parsley DC, Wei SJ (1996) Convergence to the law of one price without trade barriers or currency fluctuations. Quarterly Journal of Economics 111:1211-1236

Pflüger M, Russek S (2013) Business conditions and exit risk across countries. Open Economies Review 24:963-76

Quiggin J (1994) Regret theory with general choice sets. Journal of Risk and Uncertainty $8: 153-165$

Russ KN (2007) The endogeneity of the exchange rate as a determinant of FDI: a model of entry and multinational firms. Journal of International Economics 71(2):344-372

Sandmo A (1971) On the theory of the competitive firm under price uncertainty. American 
Economic Review 61:65-73

Schmitz M (2010) Financial markets and international risk sharing. Open Economies Review 21: 413-31

Starmer C, Sugden R (1993) Testing for juxtaposition and event-splitting effects. Journal of Risk and Uncertainty 6:235-254

Steil B (1993) Corporate foreign exchange risk management: a study in decision making under uncertainty. Journal of Behavioral Decision Making 6(1):1-31

Sugden R (1993) An axiomatic foundation of regret. Journal of Economic Theory 60:159180

Wong KP (2011) Regret theory and the banking firm: the optimal bank interest margin. Economic Modelling 28:2483-2487

Wong KP (2012) Production and insurance under regret aversion. Economic Modelling $29: 1154-1160$

Wong KP (2014) Regret theory and the competitive firm. Economic Modelling 36:172-175 